\title{
Moving Objects Detection \& Recognition using Hybrid Canny Edge Detection Algorithm in Digital Image Processing
}

\author{
R. Obulakonda Reddy, K.Reddy Madhavi, V. Nagalakshmi
}

\begin{abstract}
Recognition and detection of an object in the watched scenes is a characteristic organic capacity. Animals and human being play out this easily in day by day life to move without crashes, to discover sustenance, dodge dangers, etc. Be that as it may, comparable PC techniques and calculations for scene examination are not all that direct, in spite of their exceptional advancement. Object detection is the process in which finding or recognizing cases of articles (for instance faces, mutts or structures) in computerized pictures or recordings. This is the fundamental task in computer. For detecting the instance of an object and to pictures having a place with an article classification object detection method usually used learning algorithm and extracted features. This paper proposed a method for moving object detection and vehicle detection.
\end{abstract}

Index Terms: detection, digital image, object, recognition.

\section{INTRODUCTION}

There is a regularly expanding measure of picture information on the planet, and the rate of development itself is expanding. An information pattern appraises that in 2016 still cameras and cell phones caught more than 1.1 trillion pictures [1]. As indicated by a similar gauge, in 2020 the figure will increment to 1.4 trillion. A considerable lot of these pictures are put away in cloud benefits or distributed on the Internet. In 2014, over 1.8 billion pictures were transferred every day to the most well known stages, for example, Facebook and Instagram. [2].

Object detection and recognition method manages ordering objects into a specific class or group where object detection approach confining a particular object of need in moving video or digital computerized pictures. Each item or object class has its own specific highlights portray themselves and separate them from the others. in this way it can help to acknowledge the equivalent or comparative articles in different pictures or recordings. There are many application are exist in which object recognition and detection is applied some of them are automated vehicle parking systems, image retrieval, security, machine inspection, surveillance etc. object recognition and detection

Revised Manuscript Received on July 09, 2019.

R. Obulakonda Reddy' Dr. K.Reddy Madhavi, V. Nagalakshmi Associate Professor, CSE Dept., Institute of Aeronautical Engineering. Email: rkondareddy@gmail.com

Associate Professor, CSE Dept., Sree Vidyanikethan Engineering College, kreddymadhavi@gmail.com

Assistant Professor, CSE Dept., Mother Theresa Institute of Engineering and Technology, Email: lakshmi999.vallabhaneni@gmail.com still concern many Critical difficulties. one main problem is about power as for variety in scale, non-rigid deformations, imaging conditions and viewpoint. Large scale image retrieval is another present issue in this field. Because there need to be scaling up to thousands item classes and a large number of pictures.

In the image field object are contained and it can be recognized naturally. We can know this as object detection and this is the major problem and task in the computer field today.

\section{IMAGE PROCESSING FUNDAMENTAL}

In a paper industry in the early 1920s image processing was first introduced for submarine cable transfer the image were coded and at the receiving point by a telegraph printer image was reproduced. There had been enhancements in the framework in the mid to late 1920s. Image processing was utilized to improve the pictures of the moon taken by the Ranger 7 space test in 1964.Such strategies were utilized in the other space missions also. Image processing technique used in the medical field in 1970s. Allan M. Cormack and Godfrey N. Hounsfield mutually got The Nobel Prize for the creation of computer helped tomography in 1979. Now in these days image processing is getting increasingly more consideration due to the emphasis on two main regions [3]:

- For self ruling perception, deal with image

- For human analysis, enhancement in the image information

There are mainly two types of image processing:

Digital and analog image processing

The image processing in which analog or hard copies are required for human viewing then analog image processing is required. Examples are printout and photographs.

In digital image processing by using computer or any other devices manipulates the image in digitized way. Mainly there include three phases in digital image processing [3]. The phases are shown in figure 1:

- Low-level image processing

- Mid-level image

processing

- High-level image

Published By:

Blue Eyes Intelligence Engineering 
processing

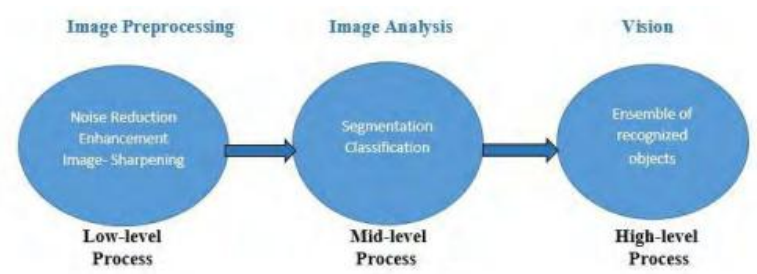

Figure 1: Low level, mid level and high level phases of Digital Image Processing

Architecture of fundamental image processing is shown in figure 2 as below:

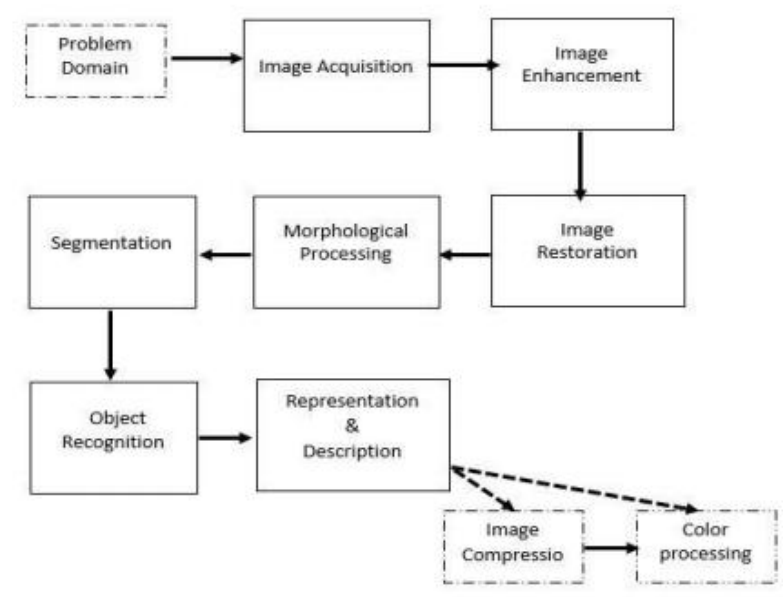

Figure 2: Fundamental Image Processing architecture

\section{CHALLENGES OF OBJECT DETECTION AND TRACKING}

In object detection general sense involves assessing the area of a specific part in progressive casings in a video grouping. Detect an object efficiently is a very hard work, especially when the object continuously change its shape, size area and direction or object have a complicated structure. In the past few decades to find the object in a specific video frame there are many methods and algorithms have been introduced by many scientists. But each method or algorithm has their own disadvantages and preferences. Any object detection algorithm has their errors and that can cause a drift. So there is need of a better algorithm that can reduce the drift to detect the accurate object over the time allotment of the application.

The main problem in object detection of a video tracker is coming when the background is same as the target object or any other object. This is known as clutter.

\section{METHOD USED FOR DETECTION OF MOVING OBJECT}

In an image sequence it is valuable to consider the different instances of movement discernible and many essential similar applications. The issues to be illuminated range from negligible detection of a moving item to examining the related movement and state of numerous moving object. In this research work we examine the four cases. To be interchangeable with the term observer we utilize the term camera. The moving object detection methods are shown in figure 3 as below:

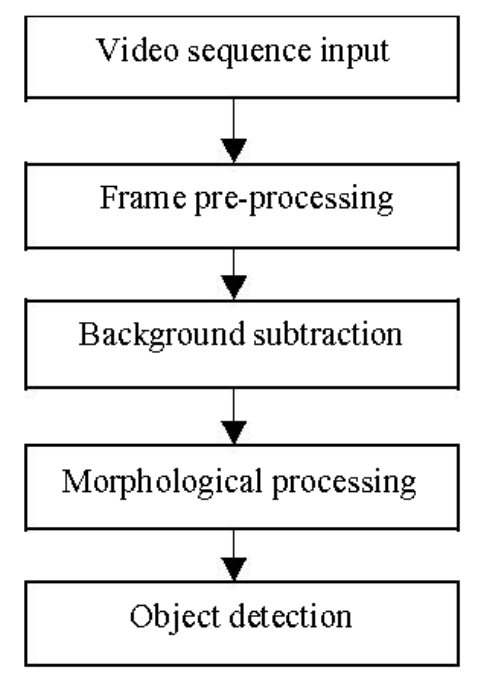

Figure 3: Moving object detection steps

In the first case the static sensor look at a moderately steady background. The moving object in that background changes to the image pixels related with the object. By detecting these pixels we can get the shape, speed and path of that object. This type of sensor commonly used in the homes where it is used for automatically switches on the light and detect the critical movement which may be because of the member of the house or to an unwelcome interloper. This type of sensor ordinarily utilized for wellbeing and safety. These types of sensor can likewise be utilized in assembling to recognize the nearness of a section encouraged into a workspace or in rush hour gridlock control frameworks that distinguish moving vehicles.

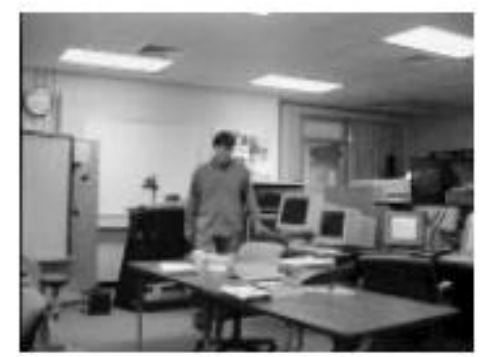

Figure 4: A person comes in an already unoccupied workspace 


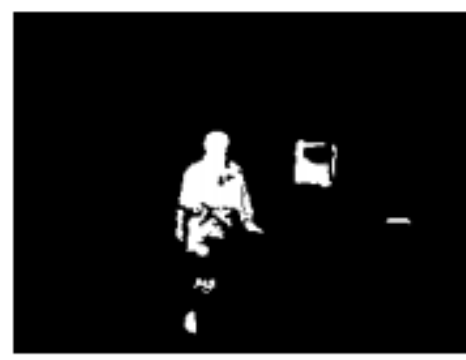

Figure 5: image subtraction reveals changed regions where the person occludes the background and at the door and a CRT.

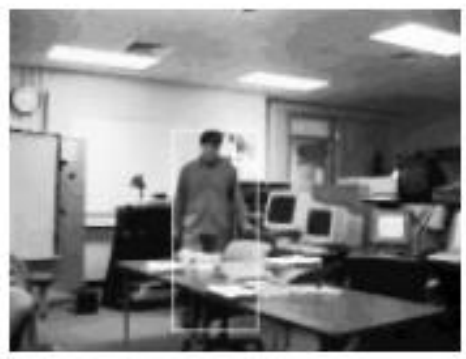

Figure 6: The change because of the individual is esteemed critical while the other two are normal and consequently overlooked

A gazing camera can likewise give information to investigation of the developments of one or a more objects. To create a direction or way the moving object must be followed after some time, this can show the conduct of that object. For example a camera can be utilized to figure out the behavior of a person in a workplace or in a lobby. A few cameras can be utilized to create various perspectives on a similar item, $\mathrm{n}$ this way empowering the calculation of ways in 3D. These types of methods are used for continually analyzing the behavior of a patient in rehabilitation center or to check the motion of an athlete. There are many other applications are there to used these types of camera.

A camera that is moving can change the images because of its own movement, regardless of whether the $3 \mathrm{D}$ condition is constant. There are many uses of this motion, This type of camera help to provide a wider view of a scene we can say this panoramic view. It might make a bigger number of perceptions of the environment. Furthermore because the images of the close object are change quicker than the image of remote articles, it can accommodate calculation of relative profundity of objects. At the third point it can accommodate discernment/estimation of the 3D state of adjacent objects. As similar to binocular sound system the various perspectives take into account a triangulating calculation. In preparing or investigating video or $\mathrm{lm}$ content, it is frequently critical to recognize focuses in time when the camera is zoomed or panned: for this situation, we may not be keen on the substance of the scene yet rather in the way where the scene was seen.

In moving sensors and scenes the most troublesome movement issues is to having such a significant number of moving object it is hard to recognize any consistent foundation. Such a case emerges with a robot vehicle exploring through huge traffic. Another fascinating case in order to follow a few moving objects in the workspace utilize a few conveying cameras to mention correspondences in their objective facts.

Detect changes between two images

Input $I_{t}[r, c]$ and $I_{t-\delta}[r, c]$ : two monochrome input images taken $\delta$ seconds apart Input $\tau$ is an intensity threshold.

$I_{\text {out }}[r, c]$ is the binary output image; $\mathbf{B}$ is a set of bounding boxes.

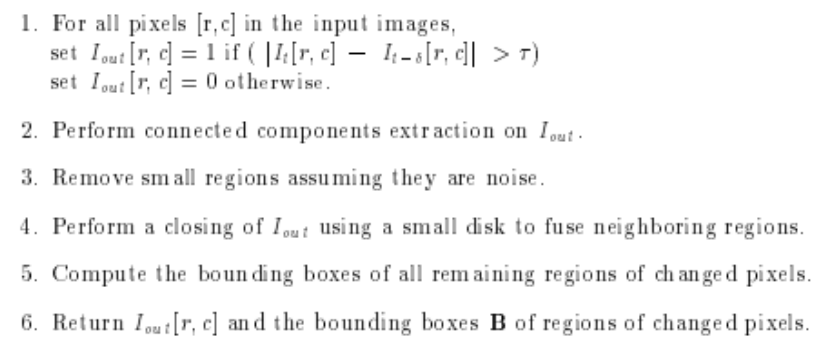

Algorithm 1: Detection of change via image subtract

\section{VEHICLE DETECTION AND TRACKING}

Going on vehicles has turned into a need rather than extravagance now in these days. Anyway simultaneously, number of passing because of the vehicular mishaps is additionally expanding every year. Overall vehicles mishaps impacts each year between 20 to 50 million individuals [3]. In this manner, building up a propelled driver help framework is ending up increasingly significant. A great deal of research is continuing building up a vision based vehicle location framework [4] [5].The fundamental objective of vision based vehicle discovery frameworks is to precisely and proficiently distinguish hazardous conditions [6]. Generally mishaps can be stayed away from by recognizing paths [7], person on foot [8] and close-by vehicles [9].

\section{VEHICLE DETECTION USING CANNY EDGE DETECTION}

Canny Edge detection algorithm is created by John F. Watchful in 1986. To detect or recognize large number of edges in an image the canny edge detector is uses multi stage algorithm. The canny edge detector is used as edge detection operator. Canny likewise created a computational hypothesis of edge recognition clarifying why the procedure works.

The Canny edge detection calculation is made out of 5 stages:

- Noise reduction;

- Gradient calculation;

- Non-maximum suppression;

- Double threshold;

- Edge Tracking by Hysteresis. 
After applying these steps, we will be able to get the following result:

\section{Noise Reduction}

Since the science required behind the scene are principally founded on subordinates (cf. Stage 2: Gradient figuring), edge detection results are effected through noise of image. One way to get rid of the noise on the image is by applying Gaussian blur to smooth it. To do so, image convolution technique is applied with a Gaussian Kernel $(3 \times 3,5 \times 5,7 x 7$ etc...). The kernel size depends on the expected blurring effect. Basically, the smallest the kernel, the less visible is the blur. In our example, we will use a 5 by 5 Gaussian kernel. Essentially, the littlest the kernel, the less noticeable is the blur.

\section{Gradient Calculation}

With the help of the edge detection operators the gradients calculators detects the intensity of the edge and direction of the edge by evaluate the gradient of the image.

\section{Non-Maximum Suppression}

Preferably, the end image ought to have slender edges. In this manner, we should perform non-most extreme concealment to disperse the edges.

The rule is straightforward: the calculation experiences every one of the focuses on the gradient intensity matrix and finds the pixels with the most extreme incentive in the edge directions.

\section{Double threshold}

The double threshold step goes for recognizing 3 sorts of pixels: non-relevant, weak and strong.

- Strong pixels will be pixels that have a power so high that we are certain they add to the last edge.

- Weak pixels will be pixels that have a power esteem that isn't sufficient to be considered as solid ones, however yet not little enough to be considered as non-significant for the edge location.

- Other pixels are considered as non-important or non relevant for the edge.

\section{Edge Tracking by Hysteresis}

The hysteresis used to convert the weak pixel in the strong pixels and it is based on the outcome of the threshold.

\section{RESULT}

The research work used to detect the vehicles in given image is shown below:

The processing methodology is shown in the figures below.

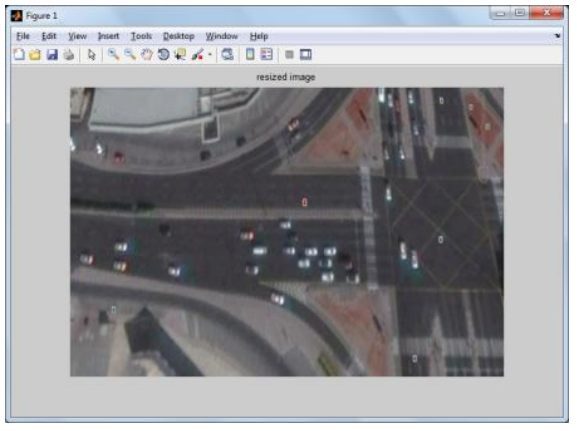

Figure 7: reference image

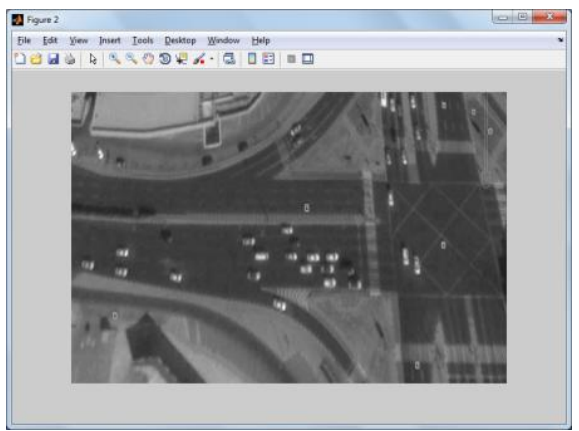

Figure 8: RGB to gray scale

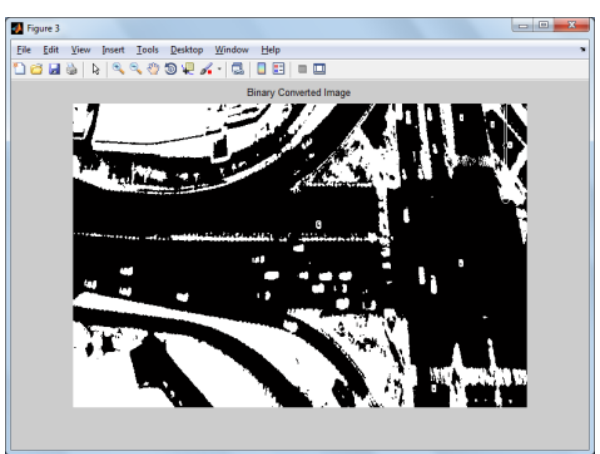

Figure 9: Binary Converted Image

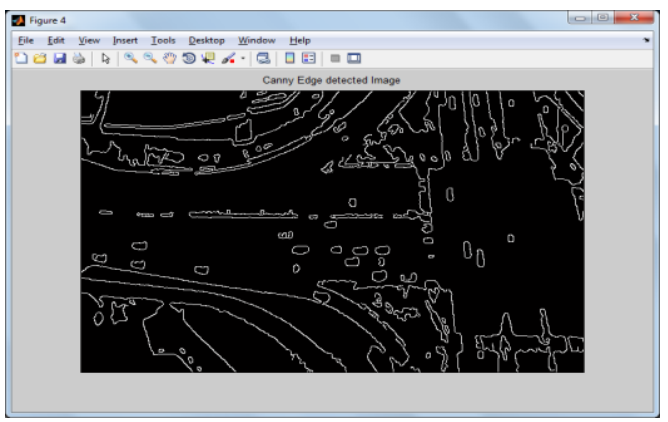

Figure 10: canny edge detection 


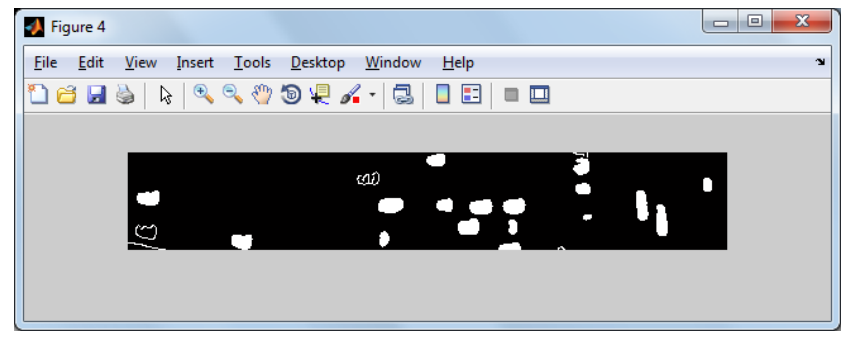

Figure 11: croped area.

The figure above shows the detection of objects. Further the experimental results are shown in the table1 below:

TABLE 1: EXPERIMENTAL RESULTS

\begin{tabular}{|c|c|c|c|c|}
\hline \multirow{2}{*}{ Vehicles } & \multicolumn{2}{|c|}{ Existing Work } & \multicolumn{2}{c|}{ Proposed Work } \\
\cline { 2 - 5 } & $\begin{array}{c}\text { No. of } \\
\text { Objects }\end{array}$ & $\begin{array}{c}\text { Accuracy } \\
\text { in } \\
\text { Percentag } \\
\text { e }\end{array}$ & $\begin{array}{c}\text { No. of } \\
\text { Object } \\
\text { s }\end{array}$ & $\begin{array}{c}\text { Accuracy } \\
\text { in } \\
\text { Percentag } \\
\text { e }\end{array}$ \\
\hline $\begin{array}{c}\text { Moving } \\
\text { Vehicle }\end{array}$ & 25 & 85 & 25 & 91 \\
\hline $\begin{array}{c}\text { Crowdy } \\
\text { Area }\end{array}$ & 78 & 79 & 78 & 84 \\
\hline $\begin{array}{c}\text { Single } \\
\text { Vehicle }\end{array}$ & 1 & 100 & 1 & 100 \\
\hline
\end{tabular}

\section{REFERENCES}

1. Infotrends - how long does it take to shoot 1 trillion photos? http: //blog.infotrends.com/?p=21573. Accessed: 2017-06-20.

2. Kpcb internet trends report 2014. http://www.kpcb.com/blog/ 2014-internet-trends. Accessed: 2017-06-20.

3. World Health Org., "World Report on Road Traffic Injury Prevention," [Online].Available:http://www.who.int/violence_injury_prevention/publi cations/road_traffic/world_report/f ctsheets/en/index.html.

4. Z. Sun, G. Bebis, and R. Miller, "On-Road Vehicle Detection Using Evolutionary Gabor Filter Optimization,” IEEE Transactions on Intelligent Transportation Systems, vol. 6, pp. 125-137, 2005.

5. Z. Sun, G. Bebis, and R. Miller, "On-Road Vehicle Detection: A Review," IEEE Transactions on Pattern Analysis and Machine Intelligence, vol. 28, p 694-7112006.

6. M. M. Trivedi, T. Gandhi, and J. McCall, "Looking-in and looking-out of a vehicle: Computer-vision-based enhanced vehicle safety," IEEE Trans. Intell. Transp. Syst., vol. 8, no. 1, pp. 108-120, Mar. 2007.

7. J. McCall and M. M. Trivedi, "Video-based lane estimation and tracking for driver assistance: Survey, system, and evaluation," IEEE Trans. Intell. Transp. Syst., vol. 7, no. 1, pp. 20-37, Mar. 2006.

8. T. Gandhi and M. M. Trivedi, "Computer vision and machine learning for enhancing pedestrian safety," in Computational Intelligence in Automotive Applications. Berlin, Germany: Springer-Verlag, May 2008 pp. 59-77.

9. T. Gandhi and M. M. Trivedi, "Vehicle surround capture: Survey of techniques and a novel Omni video based approach for dynamic panoramic surround maps," IEEE Trans. Intell. Transp. Syst., vol. 7, no. 3, pp. 293 308, Sep. 2006. 